\title{
Stellate cells and mesenchymal stem cells in benign mammary stroma are associated with risk factors for breast cancer - an observational study
}

Björn Logi Isfoss ${ }^{1,2,3^{*}}$, Bo Holmqvist ${ }^{1,4}$, Elin Sand ${ }^{4}$, Johan Forsell ${ }^{4}$, Helena Jernström ${ }^{1}$ and Håkan Olsson ${ }^{1,5}$

\begin{abstract}
Background: It is not known whether stromal cells in benign breast tissue can mediate risk of breast cancer. We recently described aldehyde dehydrogenase $1 \mathrm{~A} 1(\mathrm{ALDH} 1)$ positive $(+)$ cells in morphologically normal breast stroma of premenopausal women, and the data indicated that their distribution is associated with clinical risk factors for breast cancer. The aim of the present study was to define the identities of these cells using histologic and immunohistologic methods, and to investigate associations between those cells and hormonal and genetic risk factors in pre- and postmenopausal women.
\end{abstract}

Methods: Stroma of morphologically normal tissue was analyzed in samples from 101 well-characterized women whose breasts had been operated. Morphology and immunolabeling were applied to determine cell identities based on the putative stem cell markers ALDH1 and stage-specific embryonic antigen-3 (SSEA3), and immunophenotypes indicating mast cells or stellate cells. The results were compared with the patients' risk factors using regression analysis (two-tailed).

Results: ALDH1+ round/oval cells were associated with low parity in BRCA1/2 carriers $(p=0.022)$, while in non-BRCA1/2carriers they were negatively associated with nulliparity $(p=0.057)$. In premenopausal women ALDH1+ round/oval cells were associated with family history $(p=0.058)$. SSEA3+ round/oval cells were morphologically and immunohistologically consistent with multilineage stress-enduring (Muse) cells, and these cells were independently associated with the breast cancer risk factors low parity $(p=0.015)$, family history $(p=0.021)$, and hormone use after menopause $(p=0.032)$. $\mathrm{ALDH} 1+$ spindle-shaped/polygonal cells were immunohistologically consistent with stellate cells, and were negatively associated with family history of breast cancer $(p=0.001)$.

Conclusion: This study identified novel stromal cell types in benign breast tissue that have a potential for stratifying women for breast cancer risk.

Keywords: Breast, Breast cancer, Cancer risk, Familial cancer, Stem cells, Histology, Aldehyde dehydrogenase, BRCA1, BRCA2, Immunohistochemistry

\footnotetext{
* Correspondence: isfoss@mac.com

1Department of Clinical Sciences, Lund, Division of Oncology and Pathology, Lund University, Lund, Sweden

${ }^{2}$ Department of Pathology, Skane University Hospital, Lund, Sweden

Full list of author information is available at the end of the article
} 


\section{Background}

Nulli- and oligoparous women are at increased risk of developing breast cancer [1-3]. The risk is also higher for postmenopausal women who have received hormone therapy [4-7] and is probably at a similar level for those using the contraceptive pill [8-10]. Hereditary factors account for $5-10 \%$ of breast cancers, of which only 20 $25 \%$ are attributed to known genes such as BRCA1, $B R C A 2$, and TP53 [11]. It is has not been determined whether specific cell types in benign breast stroma are associated with susceptibility to breast cancer. The aim of the present study was to identify stromal cells in benign breast tissue and ascertain whether these cells are mediators of risk.

Most studies of cells in relation to mammary oncogenesis have focused on epithelial cells, whereas the importance of stromal stem cells is poorly understood. Also, the majority of oncogenesis-related studies of breast tissue have been performed on mechanically or chemically dissociated cells and thus have had no histological reference. Furthermore, in light of the beneficial effects of early cancer diagnosis, it might be advantageous to screen healthy women for the risk of breast cancer by performing core biopsies, a type of test that could be based on immunohistologic identification of specific epithelial or stromal cells. For these reasons, we conducted the present study to elucidate the identities of different types of stromal cells in histologically normal female breast tissue, and also to determine whether those cells are associated with clinical risk factors for breast cancer. We hypothesized that the population of round or oval-shaped ( $\mathrm{r} / \mathrm{o})$ aldehyde dehydrogenase 1 A1 positive (ALDH1+) cells in the stroma of terminal duct-lobular units (TDLUs) includes mesenchymal stem cells, and that the population of ALDH1+ spindle-shaped or polygonal (s/p) cells in the same location includes stellate cells.

Considering that anti-cancer therapy is now being designed to target stem cells [12], it is essential to map the normal histological distribution of stem markerpositive cells. Both benign stem cells and cancer stem cells in breast tissue have been reported to express ALDH1 [13, 14]. ALDH1 is a member of an enzyme family that contributes to maintaining cells intact via the detoxification of aldehydes [15], promotes cell differentiation, and converts vitamin A to its physiologically active form retinoic acid [16]. Previous studies have indicated that ALDH1 protein expression is scarce in stroma of breast carcinoma, and when present it is associated with favorable patient survival $[17,18]$.

In our earlier investigations of benign female breast tissue we used morphological and immunohistochemical methods to demonstrate that ALDH1+ cells are ductal, ductular, or stromal, and have no detectable proliferative activity [14], and also found that such cells are associated with established risk factors for breast cancer [19, 20]. Two types of ALDH1
+ stromal cells were morphologically identified, which we designated r/o cells and s/p cells. Correlating those cells with breast cancer risk factors specifically in premenopausal women showed that having a low number of ALDH1+ CD44+ CD24- r/o cells in the stroma of TDLUs was associated with family history of breast cancer, and having a low number of ALDH1+ CD44- CD24- s/p cells was associated with the breast cancer risk factor nulliparity [20]. Conversely, a high number of ALDH1+ cells in ductular epithelium was associated with the same risk factors and also with genetic risk factors for breast cancer [19]. These findings indicate that different types of stromal ALDH1+ cell types play disparate roles, and also suggest that the histological location and cell-specific identity of these and other immunophenotypic cells can provide an important basis for an improved assessment of the risks of developing breast cancer.

The morphologies the ALDH1+ r/o and s/p stromal cells that we recently described in benign breast tissue correspond morphologically to mast cells and mesenchymal stem cells [21, 22], and fibroblasts/fibrocytes and stellate cells [23-26], respectively. The last mentioned cell type is present in a wide range of tissues, and is also called the vitamin A-storing cell [23]. In previous studies $[14,20]$, we described numerous ALDH1+ s/p cells in TDLU stroma that are thin and elongated in shape and display in some tissue planes triangular cell bodies. We also demonstrated that ALDH1+ s/p cells are negative for the following: the muscle protein marker smooth muscle myosin heavy chain (SMMHC), the proliferation marker Ki-67, the pan-leukocyte marker CD45, and the epithelial cell markers Cam5.2 and E-cadherin [14]. Furthermore, the morphology and high ALDH1 expression of ALDH1+ s/p cells, along with the ubiquitous presence of these cells in subepithelial locations, suggest that they correspond to stellate cells. Stellate cells contain substantial amounts of ALDH1, an enzyme that is critical for the synthesis of retinoic acid [16].

These observations prompted us to identify ALDH1+ stromal cells that are associated with risk factors for breast cancer. To this end we applied in situ protein-detecting methods using markers for stemness and cell differentiation in morphologically normal breast tissue. Quantitative data were then compared with data from patient records concerning the presence or absence of specific hormonal and genetic breast cancer risk factors.

The cell types described here are novel for breast tissue, and if the results are validated in an independent cohort they may provide a basis for a future biopsy-based test that can stratify women with regard to the risk of breast cancer.

\section{Methods}

The study was done by applying histoloic and immunohistologic methods on benign tissue from women that 
were selected into the study for having been operated for benign and malignant conditions of the breast. The findings were compared with clinical data for examining possible assocations between cell types and risk factors for breast cancer.

\section{Clinical material}

The tissue material used in this investigation consisted of formalin-fixed and paraffin-embedded (FFPE) breast specimens from the patient groups shown in Fig. 1. Only histologically benign tissue that was free from epithelial atypia or hyperplasia was studied. The tissue samples were obtained during breast operations performed on women who had received care for breast cancer or for $B R C A 1 / 2$ carrier status, or had undergone reduction plastic surgery, at Skåne University Hospitals (Lund, Malmö, Helsingborg, Kristianstad) during the period 1983 to 2010. Clinical and immunohistologic data on the same set of patients have been used in a previous study [19].

One hundred twenty-six patients belonging to different clinical categories were randomly selected: 30 with breast cancer, 61 diagnosed with a deleterious $B R C A 1$ or $B R C A 2$ mutation, and 35 who had undergone reduction mammoplasty (members of a previously reported cohort [27]). Patients were excluded as follows: two due to prior neoadjuvant therapy; five because all tissue was lost from archive; 18 because only a limited number of histologically normal TDLUs $(<10)$ were present in any one tissue block by means of microscopy of the original slides stained with hematoxylin and eosin $(H \& E)$. The tissue block from each patient that contained the largest number of histologically normal glandular breast tissue was used in the analyses. For each of the selected patients, the median area of tissue section that was finally analyzed was 255 (range 48-621) sq. $\mathrm{mm}$.

The 101 patients who were found eligible for tissue analysis had been diagnosed as follows: 49 with no breast cancer, four with in situ carcinoma (ductal and/or lobular), 40 with invasive ductal carcinoma, seven with invasive lobular carcinoma, and one with mixed invasive ductal and lobular carcinoma. The median age of these patients was 39 years (range 20-81 years). Sixty-two women were premenopausal at the time of surgery, and 32 were postmenopausal. The menopausal status of seven women was not known. Thirty-seven women were nulliparous, 58 were parous, and six had unknown parity. Sixty patients had a first-degree relative with breast cancer, and 27 of those subjects had a BRCA1 mutation and 22 a BRCA2 mutation. One patient had unknown family history and no confirmed BRCA1/2 mutation. The investigators were blinded for the clinical information while analyzing specimens in the laboratory.

All samples from breast operation specimens were initially evaluated using the original H\&E-stained sections. In addition to the criteria mentioned above, patient material was chosen from tissue blocks that contained the largest amount of histologically normal glandular tissue including $\geq 10$ benign TDLUs.

A limited number of randomly selected samples $(n=16)$ were used for the initial identification of specific cell types (double immunofluorescence) present in the study population, with no comparisons between patient groups. Then a total of 101 patients were used for statistic evaluations of the identified specific cell types (immunohistochemistry), with comparisons between the patient groups.

\section{Double immunofluorescence labeling}

The presence and identity of $\mathrm{r} / \mathrm{o}$ and $\mathrm{s} / \mathrm{p}$ cell types was initially performed by means of double immunofluorescence (dIF) labeling. An antibody against ALDH1 was used together with antibodies described as markers for mesenchymal stem cells, mast cells, and stellate cells: ALDH1 together with SSEA3, ALDH1 with tryptase, tryptase with SSEA3, and ALDH1 with vinculin. Archival FFPE samples of tissue types recommended by the manufacturers were used to ensure specific labeling of the cell types that were targeted in this study. The dIF labeling was evaluated as cellular presence and/or co-presence of the antigens. This

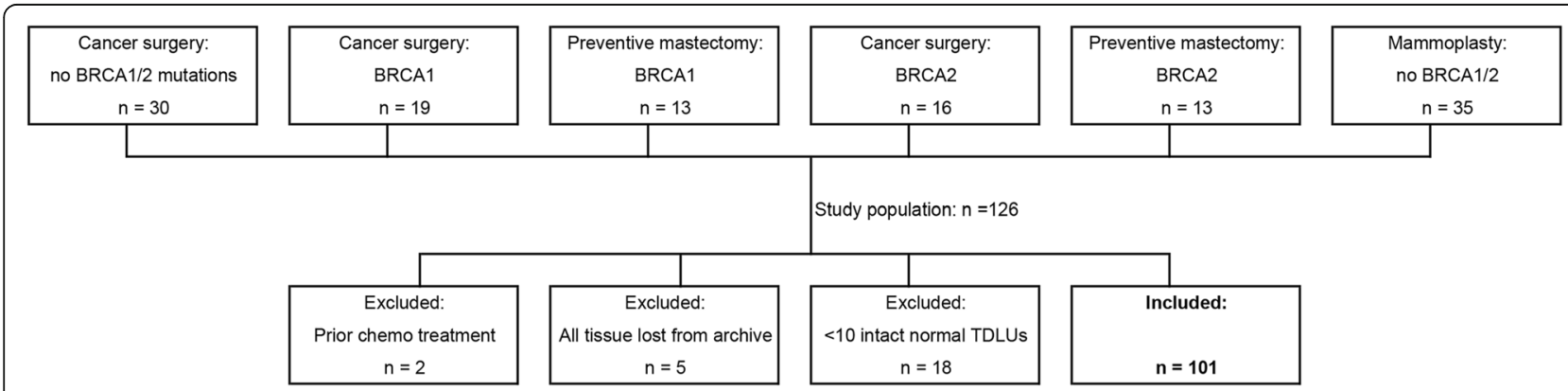

Fig. 1 Patients contributing the histologically normal breast tissue samples analyzed in the present study. Patient groups considered for inclusion in the investigation were selected based on four different breast surgery indications: cancer but no BRCA1/2 mutation; cancer and BRCA1/2 mutation; BRCA1/2 carrier status but no cancer; mammoplasty. The same set of patients was evaluated in previous studies $[14,19,20]$ 
qualitative analysis was performed on representative samples to enable morphologic identification of specific cell types expressing one or more of the investigated antigens (i.e. ALDH1, SSEA3, tryptase, and vinculin). For the double immunofluorescence labeling, 16 premenopausal women of age 31-40 years were randomly selected from the patient groups with the following breast cancer risk categories (see Table 1): nulliparous $(n=3)$, parous $(n=3)$, family history of breast cancer but no BRCA1/2 mutation $(n=4), B R C A 1$ mutation $(n=3)$, and BRCA2 mutation $(n=3)$.

For double immunofluorescence labeling, paraffin sections were heated at $60{ }^{\circ} \mathrm{C}$ for $30 \mathrm{~min}$ and then deparaffinized, starting in xylene followed by hydration in a graded alcohol series, ending with water. Thereafter, the slides were immersed in citrate acid buffer $(10 \mathrm{mM}, \mathrm{pH} 6)$ containing 0.05\% Tween 20 (both from Sigma-Aldrich, St. Louis, MO, USA). Heat-induced antigen retrieval was performed in a microwave oven $\left(3 \mathrm{~min}\right.$ at $800 \mathrm{~W}$ to $95{ }^{\circ} \mathrm{C}$, followed by $10 \mathrm{~min}$ at $200 \mathrm{~W}$ and $82^{\circ} \mathrm{C}$ ). The slides were then allowed to cool to RT before being immersed in distilled water (at $\mathrm{RT}$ ) and subsequently rinsed in phosphate-buffered saline (PBS, pH 7.4) (Medicago, Uppsala, Sweden).

The dIF immunolabeling started with incubation of sections for $30 \mathrm{~min}$ at RT in blocking solution composed of PBS containing 1\% BSA (Sigma-Aldrich) and 0.05\% Triton X100 (Applichem). Next, the sections were incubated for $16 \mathrm{~h}$ at $4{ }^{\circ} \mathrm{C}$ in a mixture of two primary antibodies raised against various antigens in different animal host species (see Table 2). Thereafter the sections

Table 1 Patient characteristics for the subset that was selected for double immunofluorescence experiments; all of age 31-

\begin{tabular}{lllll}
40 years & & & & \\
\hline Patient & Child birth(s) & $\begin{array}{l}\text { Family history of } \\
\text { breast cancer }\end{array}$ & $\begin{array}{l}\text { BRCA1/2 } \\
\text { mutation }\end{array}$ & $\begin{array}{l}\text { Pathology } \\
\text { diagnosis }\end{array}$ \\
\hline 17 & No & No & No & IDC \\
75 & No & No & No & IDC \\
91 & No & No & Nonign & Benign \\
62 & Yes & No & No & IDC \\
97 & Yes & No & No & Benign \\
59 & Yes & No & No & IDC \\
89 & Yes & Yes & IDC \\
78 & No & Yes & No & IDC \\
30 & No & Yes & No & IDC \\
83 & Yes & Yes & BRCA1 & DCIS \\
46 & Yes & Yes & BRCA1 & IDC \\
99 & Yes & Yes & BRCA1 & IDC \\
103 & Yes & Yes & BRCA2 & IDC \\
116 & No & Yes & BRCA2 & Benign \\
37 & Yes & Yes & BRCA2 & Benign \\
126 & Yes & Yes &
\end{tabular}

Abbreviations: IDC invasive ductal carcinoma DCIS ductal carcinoma in situ
Table 2 Primary antibodies used for simultaneous visualization of two antigens by double immunofluorescence or single immunohistochemical labeling of individual antigens

\begin{tabular}{|c|c|c|c|c|c|}
\hline Epitope & $\begin{array}{l}\text { Host } \\
\text { species }\end{array}$ & $\begin{array}{l}\text { Product } \\
\text { code }\end{array}$ & $\begin{array}{l}\text { Dilution } \\
\mathrm{IHC}\end{array}$ & $\begin{array}{l}\text { Dilution } \\
\text { dIF }\end{array}$ & Manufacturer \\
\hline $\begin{array}{l}\text { ALDH1 } \\
\text { A1 }\end{array}$ & $\begin{array}{l}\text { Mouse } \\
\text { (lgG) }\end{array}$ & 611,194 & $1: 1000$ & 1:100 & $\mathrm{BD}$ \\
\hline SSEA-3 & Rat (lgM) & MC-631 & 1:100 & $1: 50$ & DSHB \\
\hline Tryptase & Rabbit (lgG) & Ab134931 & $1: 2000$ & $1: 2000$ & Abcam \\
\hline Vinculin & Rabbit (IgG) & SAB4503069 & & $1: 300$ & Sigma-Aldrich \\
\hline
\end{tabular}

MC-631 (SSEA-3) was deposited to the DSHB by Solter, D. / Knowles, B.B Abbreviations: dlF double immunofluorescence, IHC immunohistochemical labeling, $B D$ Becton Dickinson Bioscience (Franklin Lakes, NJ, USA), DSHB Developmental Studies Hybridoma Bank (University of lowa, lowa City, IA, USA); Abcam (Cambridge, UK); Sigma-Aldrich (St. Louis, MO, USA)

were rinsed in PBS and incubated for $30 \mathrm{~min}$ at $\mathrm{RT}$ in a mixture of secondary antibodies made against the host species for the primary antibodies and conjugated with fluorophores for detection of the binding at different wavelengths. All antibodies were diluted in the blocking solution, and all incubations were performed in a moisture chamber. To ensure specific immunolabeling with the primary antibodies, and to exclude the risk of detecting unspecific secondary antibody binding and autofluorescence during analyses, primary antibodies were excluded from the labeling protocol for adjacent sections. Following rinses in PBS, nuclear staining was performed by incubating sections in 4', 6-diamidino-2-phenylindole (DAPI, $0.05 \mu \mathrm{M}$, Invitrogen-Thermo Fisher Scientific, Waltham, MA, USA) for $15 \mathrm{~min}$ at RT. Sections were then mounted in Fluoroshield mounting medium (Abcam, Cambridge, UK).

\section{Analyses of double immunofluorescence labeling}

To evaluate the cellular and subcellular localization of labeling by one or two primary antibodies, and to ensure primary antibody specificity and general quality of double labeling, selected dIF-labeled samples were analyzed by confocal laser scanning microscopy (Zeiss LSM710). X-Y scanning and Z-stacking (200-400-nm optical sections) were used to determine cellular co-localization of dIF labeling, or absence thereof, in morphologically defined r/o and $\mathrm{s} / \mathrm{p}$ cells. Control sections without primary antibody showed no detectable fluorophores and no or only a very low background signal, which confirmed supporting the specific binding of the primary and secondary antibodies that were used. See Additional files 1, 2, 3, 4 and 5.

All the selected tissue material from the dIF-labeled sections from 16 patients was assessed in an epifluorescence microscope (Olympus IX73, Tokyo, Japan). Sections that contained at least 10 TDLUs of good histological quality were considered evaluable. The cellular immunofluorescence labeling was evaluated by recording the visual inspection performed while switching between channels and by analyzing digital images (grabbed with a 
Olympus DP70 b/w detector) including color-coded and merged overlays of the different channels (Cell Sense software, Olympus). The use of thin sections $(5 \mu \mathrm{m})$ and the solitary appearance of stromal $\mathrm{r} / \mathrm{o}$ and $\mathrm{s} / \mathrm{p}$ cells made it possible to evaluate the morphologically identified $\mathrm{r} / \mathrm{o}$ and $\mathrm{s} / \mathrm{p}$ single cells as single or double labeled in a specific focal plane. Two of the authors (BLI and ES) conducted the dIF analyses for ALDH1 and SSEA3, ALDH1 and tryptase, tryptase and SSEA3, and ALDH1 and vinculin.

\section{Immunohistochemical labeling}

The results of the dIF experiments (see above) further supported that stromal ALDH1+ r/o cells are morphologically consistent with mesenchymal stem cells (SSEA3 + ) and/or mast cells (tryptase+), and that some ALDH1 $+\mathrm{s} / \mathrm{p}$ cells are morphologically consistent with stellate cells (ALDH1+ and vinculin+). Therefore, to enable statistic comparison with risk factors for breast cancer we performed IHC labeling of these stromal $\mathrm{r} / \mathrm{o}$ and $\mathrm{s} / \mathrm{p}$ immunophenotypic cells in the entire cohort $(n=101)$.

In the protocol used for the single IHC labeling, the sections $(5 \mu \mathrm{m})$ were initially heated to $60{ }^{\circ} \mathrm{C}$ for $30 \mathrm{~min}$ and then deparaffinized and hydrated, and processed for heat-induced antigen retrieval (as outlined above). Sections were then quenched for endogenous peroxidase activity through incubation in $0.03 \% \mathrm{H}_{2} \mathrm{O}_{2}(10 \mathrm{~min}$ at RT) and blocked for unspecific binding sites for the secondary antibodies by incubation in blocking solution (PBS containing 1\% BSA and 0.05\% Triton X100; 30 min at RT). Thereafter the sections were incubated with one primary antibody diluted in the blocking solutions for $16 \mathrm{~h}$ at $4{ }^{\circ} \mathrm{C}$ (see Table 2) and then rinsed in PBS and incubated with horseradish peroxidase (HRP)-conjugated secondary antibodies (listed in Table 3) diluted in blocking solution for $30 \mathrm{~min}$ at RT. Sections were subsequently rinsed in PBS, and the HRP conjugate was reacted for $10 \mathrm{~min}$ at $\mathrm{RT}$ in a $\mathrm{PBS}$ solution containing

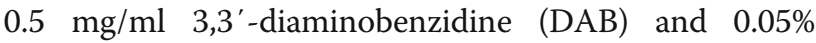
$\mathrm{H}_{2} \mathrm{O}_{2}$. The sections were then counterstained in Mayer's hematoxylin (Histolab) for $30 \mathrm{~s}$, dehydrated in a graded alcohol series ending with xylene, and mounted and coverslipped in Pertex (Histolab).

Antibody controls were made in all IHC labeling experiments by excluding incubation of the primary antibodies from the protocol on adjacent parallel sections. The specific labeling of the primary antibodies that were used was confirmed by the lack of or no comparable labeling in these control sections, which thereby further supported the specific binding of the antibodies also for IHC labeling techniques (see immunofluorescence labeling above).

\section{Analyses of Immunohistochemical labeling}

The IHC labeling was evaluated by visual inspection using microscopes equipped for bright-field microscopy (Zeiss Axioskop and Olympus IX73). The immunolabeling was assessed for individual patients by analyzing 10 40 histologically normal TDLUs. Immunolabeled $\mathrm{r} / \mathrm{o}$ and $\mathrm{s} / \mathrm{p}$ cells were recorded as present or absent, and whether they were located in TDLUs or in the border between TDLU stroma and generic stroma. The analyses were performed by two of the authors who are experienced in histology and histopathology (JF \& BLI).

The assessment of ratios for TDLUs containing any $\mathrm{r} /$ $o$ and s/p immunolabeled cells (ALDH1, SSEA3, or tryptase immunoreactive) were statistically correlated with risk factors for breast cancer according to the patients' clinical records.

\section{Statistic analysis}

The size of the studied material was not based on statistical power estimates. Correlations between identified immunophenotypes of cells, determined by microscope evaluations of dIF and IHC labeling, were tested by two-tailed Spearman's rho. The multivariate analysis using regression (logistic for discrete variables and linear for continuous variables) included the breast cancer risk factors age, family history, and parity, and additional models also included ongoing use of oral contraceptives or hormonal replacement

Table 3 Secondary antibodies used for double immunofluorescence labeling or immunohistochemistry

\begin{tabular}{|c|c|c|c|c|}
\hline Secondary antibodies for dIF & Product code & Visualization & Dilution & Manufacturer \\
\hline Donkey anti-mouse lgG (Alexa Fluor 488 conjugated) & $715-546-150$ & $\mathrm{ALDH} 1$ & $1: 200$ & $J I R$ \\
\hline Goat anti-rat IgM (Rhodamine-Red conjugated) & $112-295-020$ & SSEA-3 & $1: 200$ & $J R$ \\
\hline Donkey anti-rabbit lgG (Rhodamine red conjugated) & $711-296-152$ & Tryptase, Vinculin & $1: 200$ & $J I R$ \\
\hline Goat anti-rabbit IgG Alexa Fluor 488 conjugated & A11070 & Tryptase & $1: 200$ & Invitrogen \\
\hline Secondary antibodies for $\mathrm{IHC}$ & Product code & Visualization & Dilution & Manufacturer \\
\hline Goat anti-mouse IgG (HRP conjugated) & K4007 & $\mathrm{ALDH} 1$ & Pre-diluted & Dako/Agilent \\
\hline Goat anti-rabbit lgG (HRP conjugated) & K4011 & Tryptase & Pre-diluted & Dako/Agilent \\
\hline Goat anti-rat IgM (HRP conjugated) & $112-036-075$ & SSEA-3 & $1: 100$ & $\mathrm{JlR}$ \\
\hline
\end{tabular}

Abbreviations: dIF double immunofluorescence, IHC immunohistochemical labeling, JIR Jackson ImmunoResearch (West Grove, PA, USA); Invitrogen, Invitrogen, Thermo Fisher Scientific (Waltham, MA, USA); Dako/Agilent, Dako, Agilent Technologies (Santa Clara, CA, USA) 
therapy (HRT). $P<0.05$ was considered significant. All analyses were performed using IBM SPSS v. 20 software.

\section{Results \\ Cell-specific characterization of stromal cells by immunofluorescence labeling}

In TDLU stroma, r/o cells with ALDH1, tryptase, or SSEA3 immunolabeling exhibited morphology corresponding to that of mast cells. The results of dIF experiments using the various combinations of antibodies targeting SSEA3, ALDH1, and tryptase showed that at least one TDLU in specimens from all the investigated patients contained r/o cells displaying positivity for each of these antibodies. Double-labeled SSEA3+ ALDH1+ cells were detected in 13 of 16 patients (81\%; see Fig. 2). SSEA3 + ALDH1- cells were present in 14 of 16 patients (87\%) and SSEA3- ALDH1+ cells in 11 out of 16 patients (69\%).

ALDH1 - tryptase+ r/o cells were observed in TDLU stroma of all patients, and ALDH1+ tryptase+ r/o cells were present in TDLUs of 15 patients (94\%). ALDH1+ tryptase $-r$ /o cells were detected in TDLUs of only nine patients (56\%) (see Fig. 3).

SSEA3+ and tryptase+ double-labeled r/o cells, as well as tryptase+ SSEA3-r/o cells, were found in 15 patients
(95\%). The same number of patients had tryptaseSSEA3+ cells. Interestingly, some of the r/o tryptase+ SSEA3- and tryptase+ SSEA3+ cells exhibited extracellular immunoreactive granules, which indicates degranulation (a phenomenon commonly associated with mast cells) (Fig. 4).

Double-labeled ALDH1+ vinculin+ s/p cells were detected in all patients (Fig. 5), often intermingled with cells that were positive solely for ALDH1 or vinculin (Fig. 5). An illustration of the frequency of observed double immunophenotypes is provided in Fig. 6. A table containing data from double immunofluorescence experiments is available in the Additional files 1, 2, 3, 4 and 5.

\section{Quantitative analyses of IHC Labelings with clinical correlation}

The identities of stromal $\mathrm{r} / \mathrm{o}$ and s/p cells were analyzed by single IHC labeling of ALDH1, SSEA3, and tryptase. Samples from 90 patients were of adequate technical quality (tissue from 90 patients was non-analyzable) IHC single labeling was used for statistical correlations with clinical records. Representative images of the IHC labeling are shown in Fig. 7.
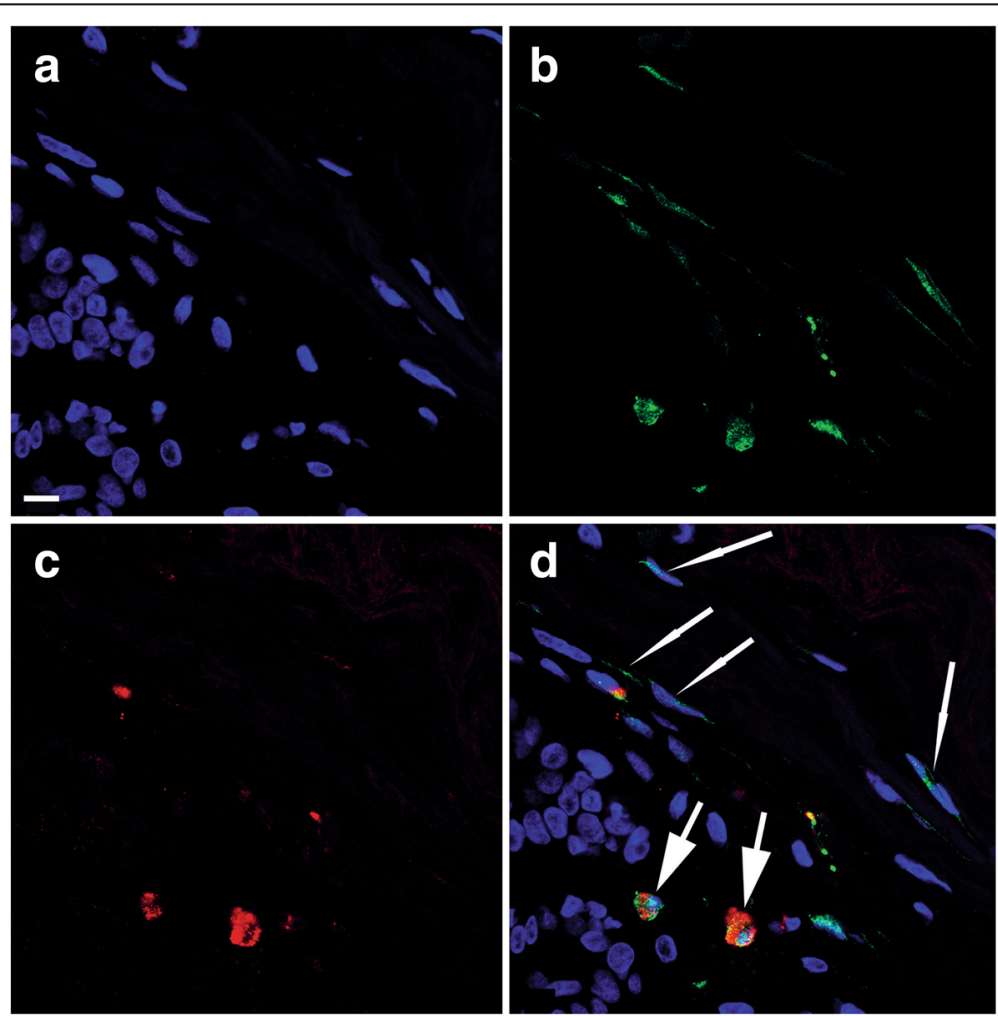

Fig. 2 Confocal images of double immunofluorescence labeling of ALDH1 and SSEA3. Scale bars in all images $10 \mu$ m. a-c Three images of the same tissue area illustrating nuclear organization and morphology: DAPI labeling blue in $\mathbf{a}$, ALDH1 labeling green in $\mathbf{b}$, and SSEA3 labeling red in c. $\mathbf{d}$ A digital composite of the three images of ALDH1 and SSEA3 immunolabeling and DAPI staining. Two double-positive ALDH1+ SSEA3+ r/O cells (thick arrows) and four single-positive ALDH1 $+s / p$ cells (thin arrows) are indicated 

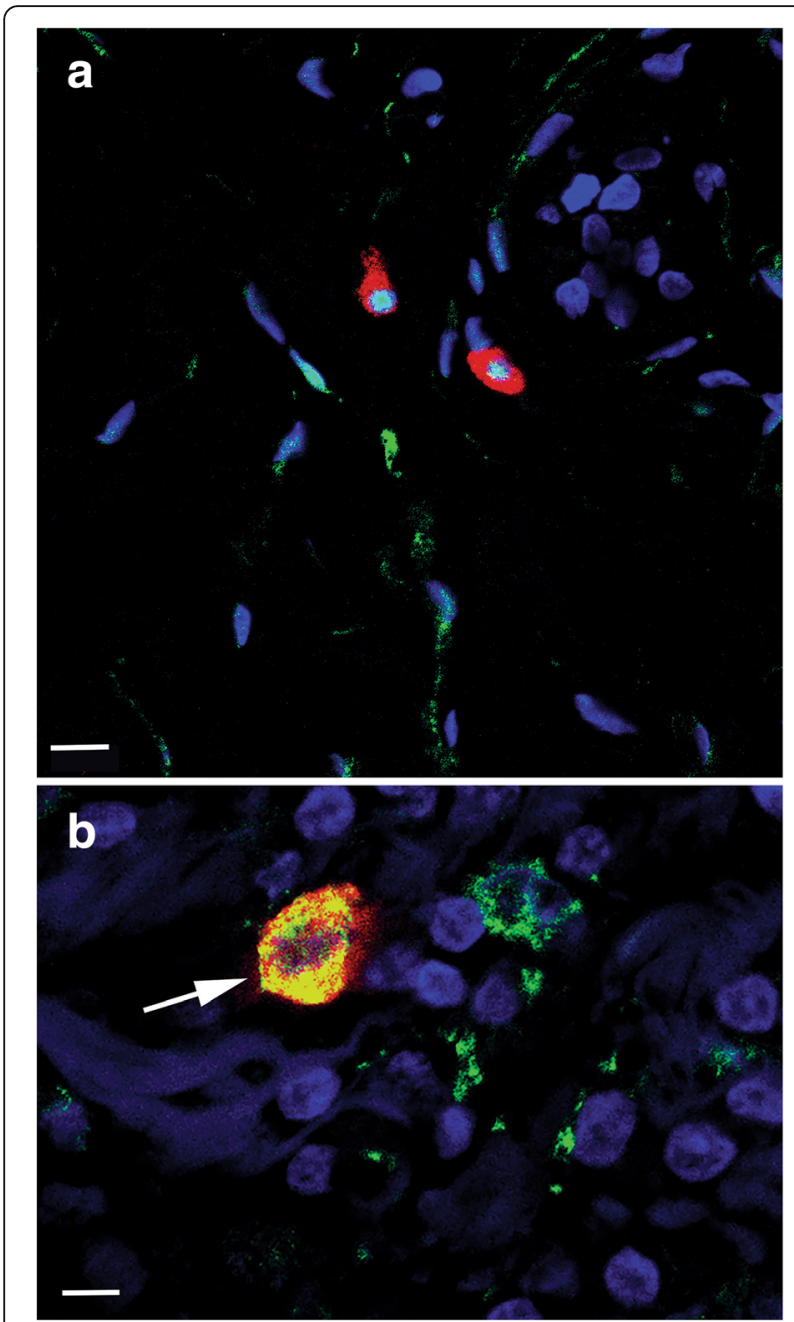

Fig. 3 Confocal images of double immunofluorescence labeling of ALDH1 and tryptase. The images are digital composites from one focal plane showing merged channels with dIF-labeled ALDH1 (green) and tryptase (red), together with nuclear staining with DAPI (blue). a Image illustrating two ALDH1- SSEA+ round/ oval cells (strong red) above scattered ALDH1+ SSEA- spindleshaped cells (green). Scale bar $10 \mu \mathrm{m}$. b Image showing an area of tissue containing one large ALDH1+ tryptase+ double-labeled r/o cell (arrow). The ALDH1 labeling (green) to the right of this cell represents parts of ALDH1+ cytoplasm from different cell types. Double labeling appears as yellow. Scale bar $5 \mu \mathrm{m}$

R/o cells were ALDH1+ in samples from $65 \%$ of the patients, and such cells were present in a median of $5 \%$ of the TDLUs. The corresponding figures for SSEA3+ r/o cells were considerably higher (99 and 43\%), and for tryptase+ r/o cells still higher (99 and 67\%). Significant correlations between the number of TDLUs containing the different r/o cell immunophenotypes were found for the following: SSEA3+ and tryptase+ for the whole patient set $(p=0.001)$; ALDH1+ and tryptase+ for the premenopausal group only $(p=0.033)$. In contrast, the correlation coefficient was very low for ALDH1+ and SSEA3+ r/o cells

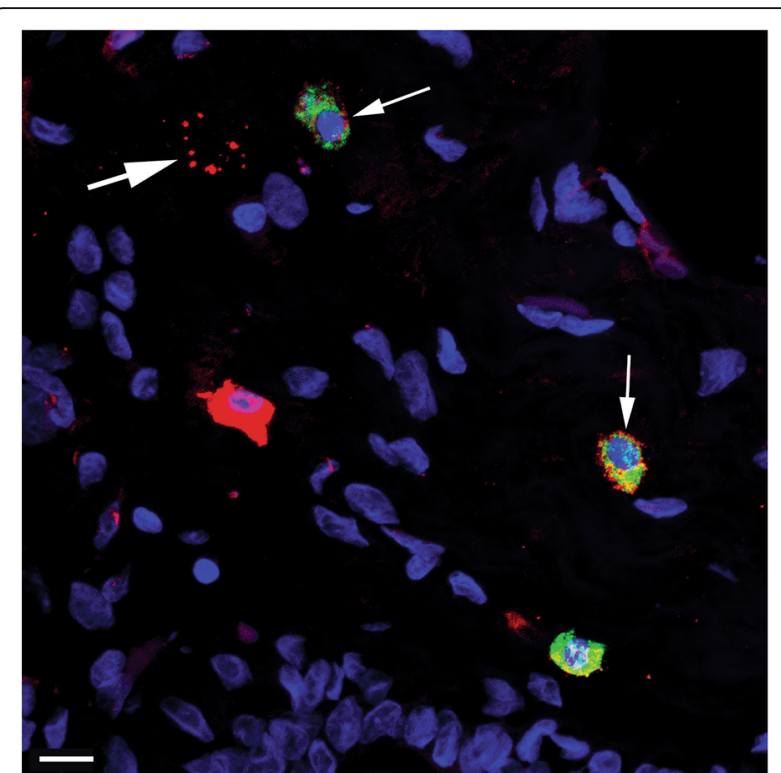

Fig. 4 A confocal image of double immunofluorescence labeling of tryptase and SSEA3. The image is a "maximum intensity" digital composite from 12 focal planes showing merged channels from dIF labeling of tryptase (green) and SSEA3 (red), together with DAPI nuclear staining (blue). Double-labeled tryptase+ SSEA3+ round/oval cells (thin arrows) and a SSEA3+ degranulated cell (thick arrow) are indicated. Scale bar $10 \mu \mathrm{m}$

(0.168), and the $p$ value indicated no statistical significance for co-presence of these two immunophenotypes in tissue samples.

Regarding ALDH1+r/o cells in TDLU stroma, the strongest statistic significance for an association with data from clinical records was found for larger numbers of these cells with decreasing parity, and only for the patient group with $B R C A 1$ or $B R C A 2$ mutations ( $p=$ 0.022). Among non-carriers, parous women had higher numbers of these cells $(p=0.057)$. Furthermore, when ongoing oral contraceptive use was included in the model, a near-significant positive association was found between high ALDH1+r/o cells and family history for premenopausal women $(p=0.058)$.

The number of SSEA3+ r/o cells associated with different risk factors for breast cancer was clearly significant. SSEA3+ r/o cells in TDLU stroma showed a positive association with a family history of breast cancer for all women $(p=0.021)$, and also for premenopausal women when the current use of oral contraceptives was included in the model $(p=0.009)$. With increasing parity, there was a significant negative association with SSEA3+ r/o cells for the total patient group $(p=0.015)$. However, when data on premenopausal patients only were analyzed and adjusted for ongoing oral contraceptive use, there was a negative association between SSEA3+ r/o cells and nulliparity $(p=0.042)$. Our results also indicated 


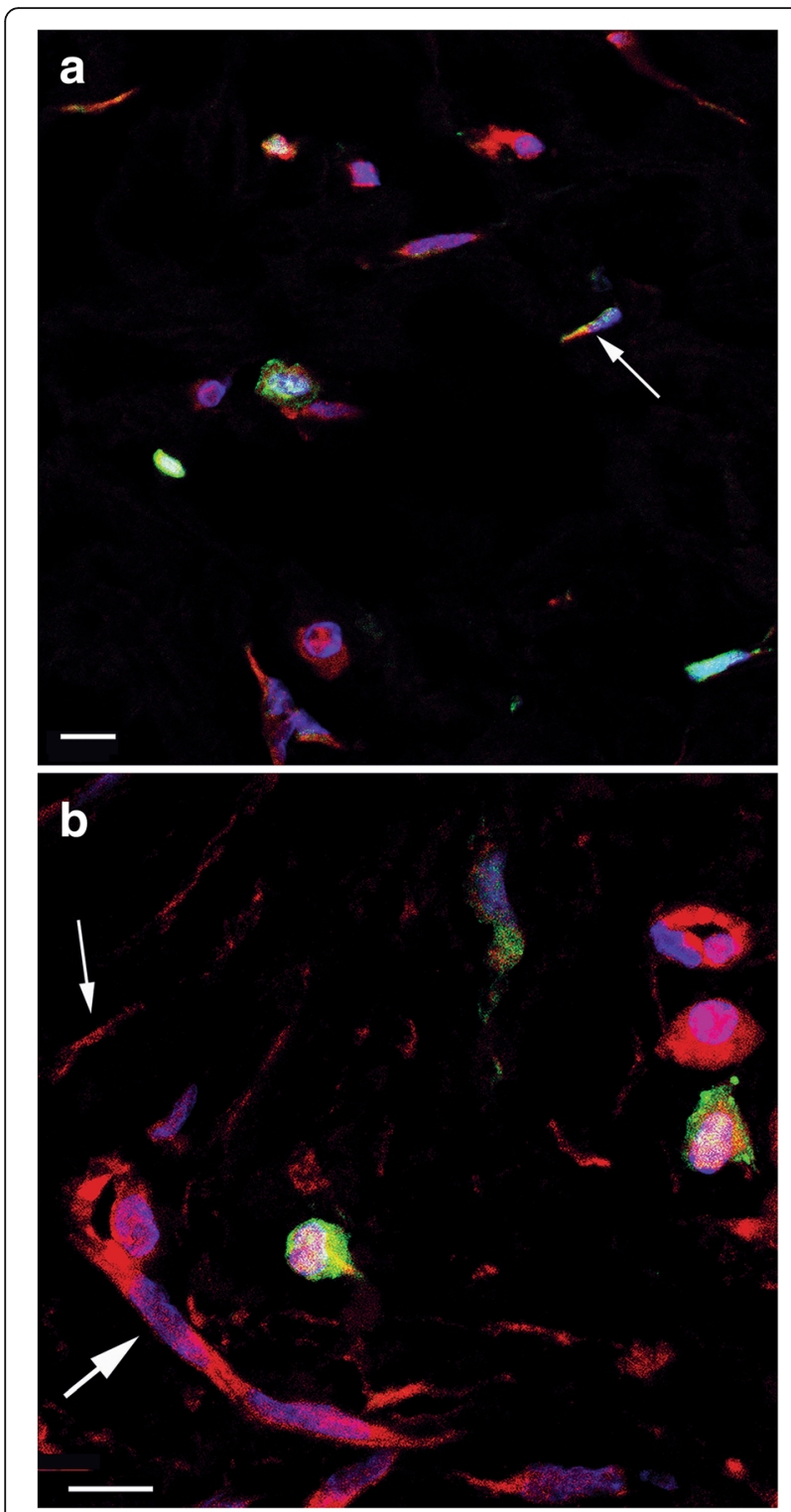

Fig. 5 Confocal images of double immunofluorescence labeling of ALDH1 and vinculin. (a and $\mathbf{b}$ ) The two images illustrate several spindleshaped and polygonal ALDH1+ (green) and/or vinculin+ (red) cells, and the variable ALDH1 and vinculin immunolabeling of these cells. Nuclei are DAPI stained (blue). A thin arrow indicates an ALDH1 and vinculin double-labeled cell (yellow) in a and an ALDH- vinculin+ cell in $\mathbf{b}$. The thick arrow in $\mathbf{b}$ indicates a capillary with vinculin+ endothelium (as reported previously [37]). Scale bars $10 \mu \mathrm{m}$

a positive association between SSEA3+ r/o cells in TDLU stroma and hormone use at the time of surgery in postmenopausal women $(p=0.032)$.

No statistically significant associations were observed between the number of tryptase+ r/o cells in TDLU stroma and risk factors for breast cancer.

ALDH $1+$ s/p cells were detected by chromogenic IHC in TDLU stroma of $92 \%$ of the patients $(83 / 90)$ and were typically present in the majority of the TDLUs (median $75 \%)$. These cells were about half as common in the TDLUs of premenopausal patients with a family history of breast cancer compared to the TDLUs of premenopausal subjects with no family history (median $46 \%$ vs. $88 \%, p=0.003)$. A reduced regression model including parity, family history, and age indicated that ALDH1+s/ $\mathrm{p}$ cells were negatively associated with family history both for all women $(p=0.001)$ and for premenopausal women $(p=0.001)$. This association was not significant when ongoing hormone use were included in the model.

No SSEA3 or tryptase labeled s/p cells were detected. The positive and negative associations identified between cell types and examined risk factors are illustrated in Fig. $6 \mathrm{~b}$.

\section{Discussion}

\section{Round/oval stromal cells}

$\mathrm{R} / \mathrm{o}$ cells in stroma were previously shown to be negative for Cam5.2 in this patient material, thus ruling out epithelial cell type [14]. In histologically normal TDLUs, co-expression in $\mathrm{r} / \mathrm{o}$ cells was revealed for all combinations of double-labeling of ALDH1, SSEA3, and tryptase in the vast majority of the specimens from the investigated patients. Of these three epitopes, only tryptase is considered to be a marker of mast cell identity, although we noted that ALDH1+ r/o cells and SSEA3+ r/o cells also exhibited mast-like morphology, including degranulation. These findings, together with significant co-presence of tryptase + cells and SSEA3+ cells, and co-presence of tryptase+ cells and ALDH1+ cells, suggests that these cell types either are, or have functions in common with, mast cells. However, there was no correlation between the presence of ALDH1 $+r /$ o cells and SSEA3+ r/o cells in the investigate material. These two r/o immunophenotypes may thus represent different cells, an interpretation that is further supported by the demonstrated differences in associations with risk factors between these two immunotypes. Furthermore, tryptase + r/o cells were not statistically associated with any of the examined risk factors, which indicates that differentiated mast cells do not play a role in the risk of breast cancer.

We have previously shown that the granular cytoplasm of ALDH1+ r/o cells strongly expresses the contractile protein marker SMMHC [14], which is compatible with a migratory quality that can be expected in bone-marrowderived cells. Importantly, the r/o cellular morphology found in the above-mentioned immunophenotypes is congruent with the pluripotent mesenchymal cells called multilineage stress-enduring (Muse) cells, with the immunophenotypic identity defined by SSEA3 positivity [21, 22]. For in situ studies, which include morphologic assessment of cell location, it is not necessary to apply the other 


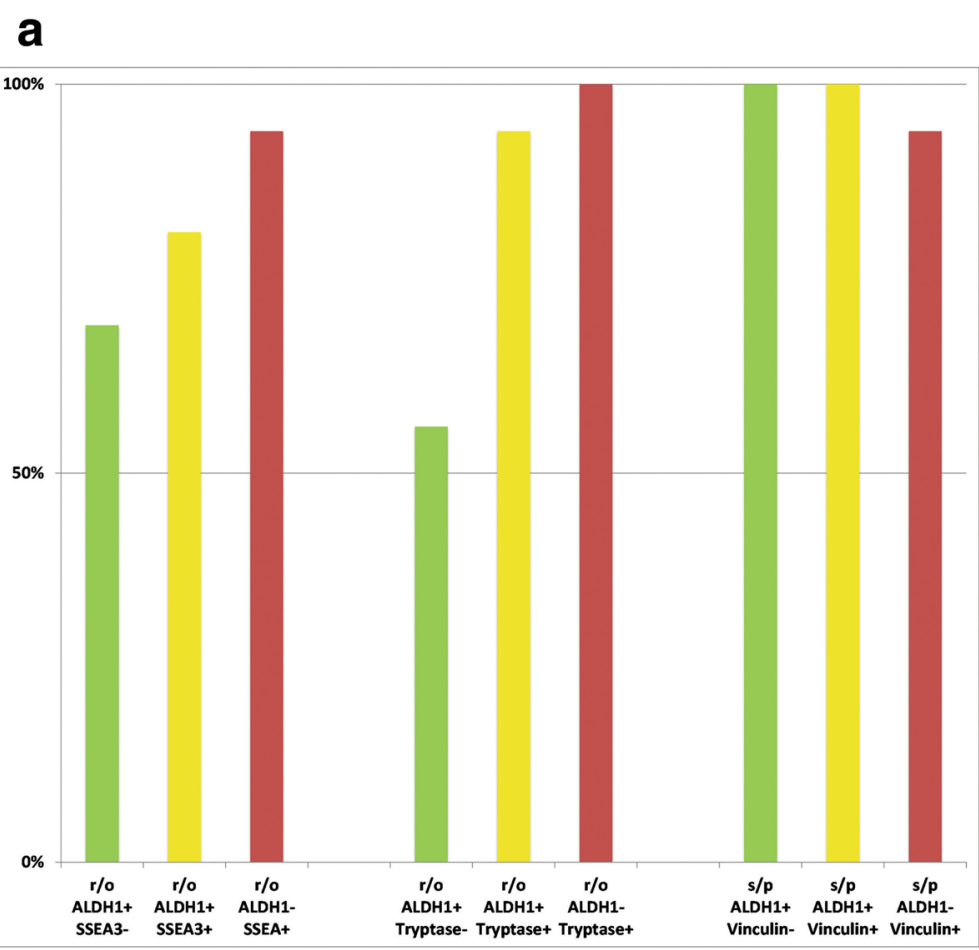

b
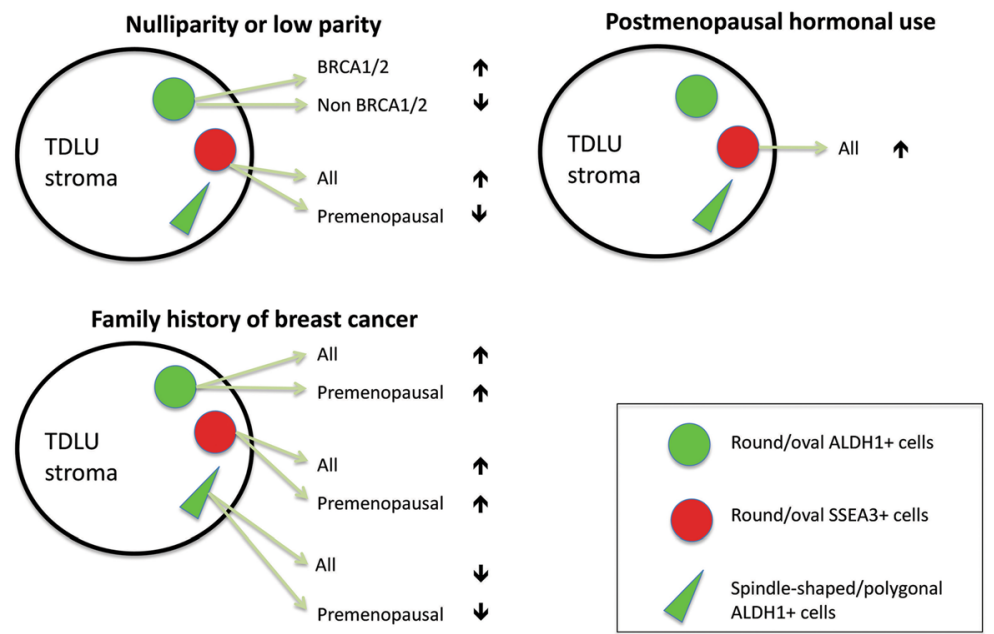

Fig. $\mathbf{6}$ a Identified stromal cell immunophenotypes detected with double immunofluorescence labeling in histologically normal TDLUs. The result is presented as the percentage of patients (Y-axis) whose specimens contained one or more $\mathrm{r} / \mathrm{o}$ and $\mathrm{s} / \mathrm{p}$ cells that were double labeled (yellow) and/or single labeled with SSEA3 or tryptase (both red), or ALDH1 (green) in histologically normal TDLU stroma $(n=16$, see also Table 1). $\mathbf{b}$ Schematic illustration of positive and negative (arrows up or down) associations between hormonal and genetic risk factors and identified cells in stroma of histologically normal TDLUs

determinant of Muse cells, the mesenchymal cell marker CD105 [21, 22]. The pluripotency of SSEA3+ cells has been confirmed in goats [28], and SSEA3+ cells in colorectal mucosa have been localized to stroma but not to epithelium [29]. In contrast, two studies have reported that SSEA3+ stromal cells are absent in breast tissue [12, 30]. However, the mentioned observations in mammary tissue were based solely on flow cytometry (i.e., dependent on cell surface immunolabeling), and hence they do not conflict with our results or the findings outlined by Dezawa et al. [22] in which IHC demonstrated that SSEA3 is present in the cytoplasm.

According to our data, ALDH1+ r/o cells are negatively associated with parity in $B R C A 1 / 2$ mutation 

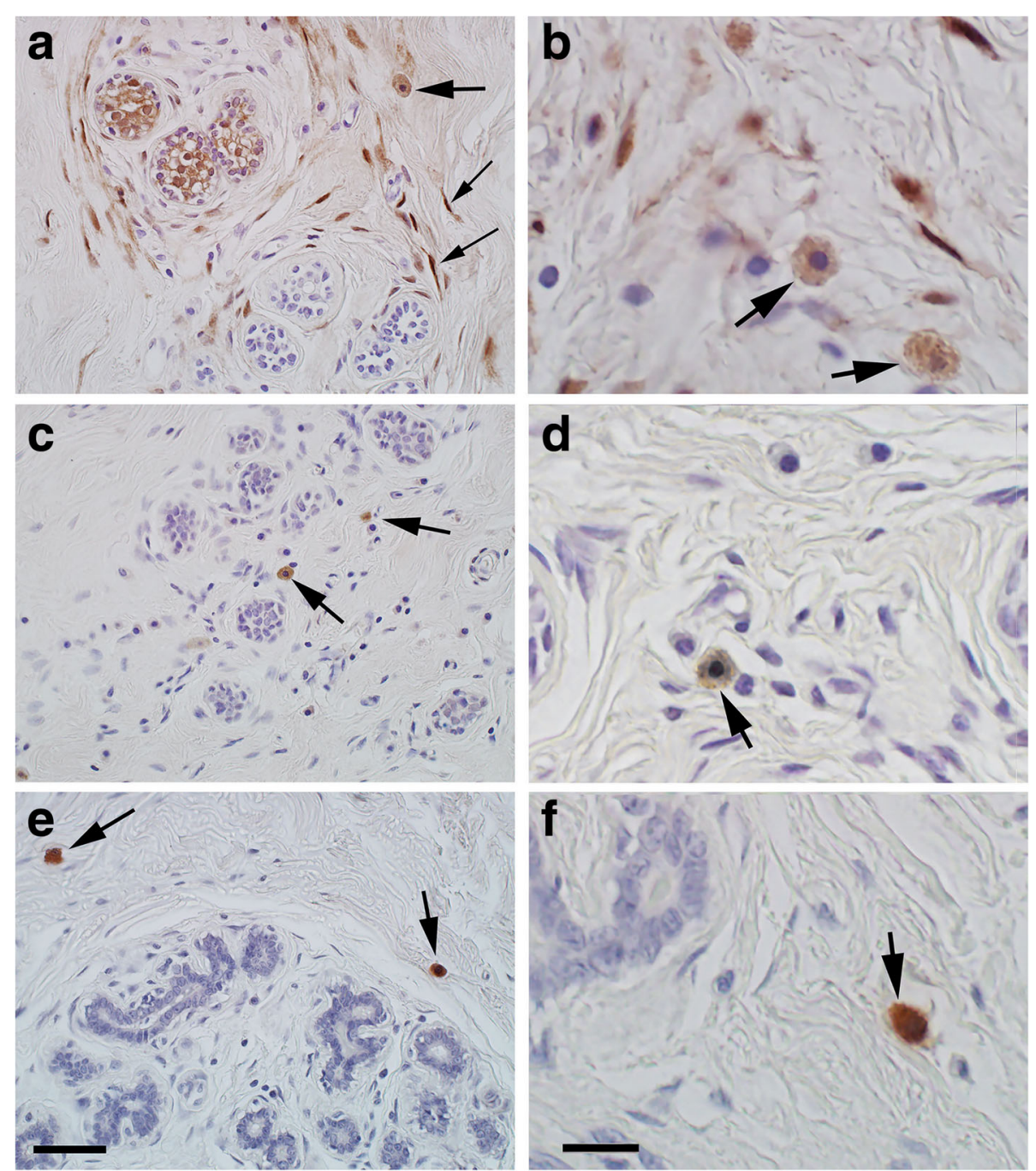

Fig. 7 Representative images demonstrating single immunohistochemical labelings performed on morphologically normal breast specimens from 90 patients. The immunolabeling was used for quantitative assessments of ALDH1, SSEA3, and tryptase r/o and/or s/p cells that were statistically evaluated in relation to patient groups. a and $\mathbf{b}$ The two identified ALDH1 immunoreactive cell types: r/o (thick arrows) and s/p (thin arrows). $\mathbf{c}$ and $\mathbf{d}$ Images showing the identified $\mathrm{r} / \mathrm{o}$ SSEA3+ cell type (arrows). e and $\mathbf{f}$ Images illustrating the identified tryptase r/o cell type (arrows). Scale bars: $50 \mu \mathrm{m}$ in $\mathbf{a}, \mathbf{c}$, and $\mathbf{e} ; 10 \mu \mathrm{m}$ in $\mathbf{b}, \mathbf{d}$, and $\mathbf{f}$

carriers but positively associated with parity in noncarriers. This is very interesting, because different numbers of pregnancies have been reported to have opposing effects on the risk of breast cancer in women carrying the BRCA mutation [31-33].

We detected an increased presence of ALDH1+ r/o cells in premenopausal women with family history of breast cancer, this was statistically near-significant and was found only after adjusting for oral contraceptives.

Our previous investigation of benign mammary stroma indicated that low presence of ALDH1+ CD44+ CD24$\mathrm{r} / \mathrm{o}$ cells in premenopausal women is marginally correlated with a family history of breast cancer, but that this does not apply to ALDH1+ CD44- CD24- cells [20]. Although findings in the current study were not statistically significant for ALDH1+r/o cells, they do suggest that the presence of such cells in histologically normal TDLUs is affected by hormones.

In the current study we found that SSEA3+r/o cells were significantly associated with family history when all patients were analyzed together, and this association was even stronger when premenopausal patients were investigated separately. SSEA3+ r/o cells were also associated with low parity, and a weaker negative association was noted for the premenopausal group but only when the data were adjusted for ongoing use of oral contraceptives. These apparently menopause- and contraceptive pill-dependent differences in associations between SSEA3+ r/o cells and parity strongly indicate a hormonal link and merit further investigation. 
We observed a signifiant relationship between SSEA3+ r/o cells and ongoing postmenopausal hormone use, which suggests that such treatment increases the number of mesenchymal stem cells in breast tissue. Notably, other investigators have reported more extensive stroma in hormone-treated patients [34].

Our morphologic and immunohistologic findings for SSEA3+ cells correspond to the above-mentioned Muse cells $[12,21,22]$, which supports the assumption that the $\mathrm{r} / \mathrm{o}$ stromal cell population in benign breast stroma contains mesenchymal stem cells. It is at best premature to suggest a causative mechanism between SSEA3+ r/o cells and breast cancer. Muse cells are derived from bone marrow, have powerful tissue regeneration properties [35], and increase markedly in number in circulating blood after ischemic injury [36]. Accordingly, large numbers of SSEA3+ r/o cells may reflect a microenvironment that is responding to tissue injury, which would be congruent with early oncogenesis.

\section{Spindle-shaped/polygonal cells}

The present study aimed to test the hypothesis that the ALDH1-expressing s/p cells in TDLU stroma are stellate cells, a term that refers to a specific cell type (also known as vitamin A-storing cell) $[24,26]$. In all of the investigated patients $\mathrm{s} / \mathrm{p}$ cells in the TDLU stroma co-labeled for ALDH1 and the stellate cell marker vinculin, and groups of $\mathrm{s} / \mathrm{p}$ cells exhibited variations in positivity for either or both those markers. Other researchers have reported that vinculin is efficacious in labeling resting stellate cells [37]. Thus the morphology and the immunophenotype of the $\mathrm{s} / \mathrm{p}$ cells identified in the current study are entirely consistent with stellate cells. Due to the close relationship between ALDH1 and vinculin expression in s/p cells, IHC to assess clinical correlation was performed only for ALDH1 in our investigation. The results indicated a highly significant association between low numbers of ALDH1+ $\mathrm{s} / \mathrm{p}$ cells in premenopausal women and family history of breast cancer. Correspondingly, in our recent study of mammary stroma in premenopausal women [20], low occurrence of ALDH1+ s/p cells was associated with the risk factor nulliparity.

These data linking relative lack of ALDH1+ s/p cells with breast cancer risk factors should be viewed considering that ALDH1 is an enzyme that catalyzes the production of retinoic acid (the physiological end product of vitamin A), a compound necessary for cell differentiation. It is logical to argue that lack of drivers of epithelial cell differentiation may predispose to carcinoma. In keeping with this, a recent study indicated that expression of ALDH1 in breast tumor stroma is associated with favorable outcome [18], and retinoic acid alone can trigger the differentiation of malignant cells and cause clinical remission [38].
Only a few studies have evaluated stromal cell types in normal breast tissue. One investigation distinguished two different types of elongated stromal cells with identical morphology, one present in TDLUs and the other found in generic stroma of the breast [39]. The former cell type was characterized as having a CD105 $5^{\text {high }}$ and CD26 ${ }^{\text {low }}$ immunophenotype, and only this population stimulated epithelial growth and branching from epithelial progenitor cells. The authors described this cell type as fibroblasts (i.e. morphology similar to that of stellate cells), and thus it is possible that we are describing the same cells in the present study. Regardless of whether that is the case, future research should focus on determining whether retinoic acid deficiency in subepithelial stroma is a contributing factor in mammary carcinogenesis.

\section{Conclusion}

This study describes immunohistologic characteristics of several cell types that are novel for benign human female breast tissue. According to clinical data presented here these cells are associated with genetic and hormonal risk factors for breast cancer.

\section{Additional files}

Additional file 1: Methodologic considerations. (DOCX $102 \mathrm{~kb}$ )

Additional file 2: Table S1. Results from double immunofluorescence experiments. (DOCX $42 \mathrm{~kb}$ )

Additional file 3: Table S2. Single immunohistochemistry data. Sortable by cell location and morphology, and by reduced clinical data. (XLSX $15 \mathrm{~kb}$ )

Additional file 4: Key for column names and coded content in single immunohistochemistry data table. (DOCX $13 \mathrm{~kb}$ )

Additional file 5: Supplementary Figure A representative dIF image of a tissue section analyzed in this study, here without primary antibody, demonstrating absence of nonspecific binding or background signals. Blue: histochemical nuclear staining by DAPI. (JPEG 1660 kb)

\section{Abbreviations}

ALDH1: Aldehyde dehydrogenase $1 \mathrm{~A} 1$; dIF: double immunofluorescence; FFPE: Formalin-fixed, paraffin-embedded; H\&E: Hematoxylin and eosin; IHC: Immunohistochemistry; R/o cells: Round or oval cells; S/p cells: Spindleshaped or polygonal cells; SMMHC: Smooth muscle myosin heavy chain; SSEA3: Stage-specific embryonic antigen-3; TDLU: Terminal duct-lobular unit

Acknowledgements

We would like to thank Ms. Patricia Ödman for language editing.

\section{Funding}

Funding was provided for this study indirectly as part of larger assignments by the European Research Council (Advanced Grant ERC-2011-AdG 294576-risk factors cancer) and the Swedish Cancer Society. Skåne Hospital Funds funded this study specifically. The funding bodies did not participate in design, collection, analysis or interpretation of data, or writing of the manuscript.

Availability of data and materials

Anonymized data generated or analyzed in this study are included in this article and its supplementary information files. More detailed clinical data will not be made available in order to protect the participants' identity. 


\section{Authors' contributions}

$\mathrm{BLI}, \mathrm{HO}$ and $\mathrm{BH}$ designed the study. $\mathrm{HO}$ and $\mathrm{HJ}$ provided material to the study. BLI, ES, JF and BH performed laboratory experiments and registered findings. $\mathrm{HO}$ and $\mathrm{BLI}$ performed statistic analysis of data. BLI drafted the manuscript. $\mathrm{BH}, \mathrm{HJ}$ and $\mathrm{HO}$ provided critical review of the manuscript. All authors contributed to final editing of the manuscript. All authors consent to publication of the submitted manuscript. All authors read and approved the final manuscript.

\section{Ethics approval and consent to participate}

The Research Ethics Committee for Southern Sweden (11-92, 349-00) approved the study. All participating patients provided a written informed consent for the scientific study and in the ethical approval it is stated that data should be published in such a manner that the identity of an individual patient is not revealed.

\section{Consent for publication}

Not applicable.

\section{Competing interests}

The authors declare that they have no competing interests.

\section{Publisher's Note}

Springer Nature remains neutral with regard to jurisdictional claims in published maps and institutional affiliations.

\section{Author details}

'Department of Clinical Sciences, Lund, Division of Oncology and Pathology, Lund University, Lund, Sweden. ${ }^{2}$ Department of Pathology, Skane University Hospital, Lund, Sweden. ${ }^{3}$ Department of Pathology, Telemark Hospital, Ulefossv. 55, 3710 Skien, Norway. ${ }^{4}$ ImaGene-iT, Medicon Village, Lund, Sweden. ${ }^{5}$ Department of Clinical Sciences, Lund, Division of Cancer Epidemiology, Lund University, Lund, Sweden.

\section{Received: 14 June 2017 Accepted: 19 February 2018}

Published online: 27 February 2018

\section{References}

1. MacMahon B, Cole P, Lin TM, et al. Age at first birth and breast cancer risk. Bull world Helth. Organ. 1970;43:209-21.

2. Adami HO, Hansen J, Jung B, Rimsten AJ. Age at first birth, parity and risk of breast cancer in a Swedish population. Br J Cancer. 1980;42:651-8.

3. $\mathrm{Ma} \mathrm{H}$, Henderson KD, Sullivan-Halley J, et al. Pregnancy-related factors and the risk of breast carcinoma in situ and invasive breast cancer among postmenopausal women in the California teachers study cohort. Breast Cancer Res. 2010;12:R35.

4. Collaborative Group on Hormonal Factors in Breast Cancer. Breast cancer and hormone replacement therapy: collaborative reanalysis of data from 51 epidemiological studies of 52,705 women with breast cancer and 108,411 women without breast cancer. Lancet. 1997;350:1047-59.

5. Olsson $\mathrm{H}$, Ingvar $\mathrm{C}$, Bladström A. Hormonal replacement therapy containing progestins and given continuously increases breast carcinoma risk in Sweden. Cancer. 2003;97:1387-92.

6. Million Women Study Collaborators. Breast cancer and hormonereplacement therapy in the million women study. Lancet. 2003;362:419-27.

7. Jernström H, Bendahl PO, Lidfeldt J, et al. A prospective study of different types of hormone replacement therapy use and the risk of subsequent breast cancer: the women's health in the Lund area (WHILA) study (Sweden). Cancer Causes Control 2003;14:673-680.

8. Olsson H, Möller TR, Ranstam J. Early oral contraceptive use and premenopausal breast cancer - final report from a study in southern Sweden. J Natl Cancer Inst. 1989;81:1000-4.

9. Jernström $\mathrm{H}$, Loman $\mathrm{N}$, Johannsson OT, et al. Impact of teenage oral contraceptive use in a population-based series of early-onset breast cancer cases who have undergone BRCA mutation testing. Eur J Cancer. 2005;4:2312-20.

10. Samson ME, Adams SA, Mulataya CM, et al. Types of oral contraceptives and breast cancer survival among women enrolled in Medicaid: a competingrisk model. Maturitas. 2017;95:42-9.

11. Fackenthal JD, Olopade OI. Breast cancer risk associated with BRCA1 and BRC2 in diverse populations. Nat Rev Cancer. 2007;7:937-48.
12. Cheung SKC, Chuang PK, Huang HW, et al. Stage-specific embryonic antigen-3 (SSEA-3) and BGalT5 are cancer specific and significant markers for breast cancer stem cells. Proc Natl Sci U S A. 2016;113: 960-5.

13. Ginestier $\mathrm{C}$, Hur MH, CHarafe-Jauffret $\mathrm{E}$, et al. ALDH1 is a marker of normal and malignant human mammary stem cells and a predictor of poor clinical outcome. Cell Stem Cell. 2007;1:555-67.

14. Isfoss BL, Holmqvist B, Alm P, et al. Distribution of aldehyde dehydrogenase 1 -positive stem cells in benign mammary tissue from women with and without breast cancer. Histopathology. 2012;60:617-33.

15. Marchitti SA, Brocker C, Stagos D, et al. Non-P450 aldehyde oxidizing enzymes: the aldehyde dehydrogenase superfamily. Expert Opin Drug Metab Toxicol. 2008:4:697-720.

16. Duester G. Retinoic acid synthesis and signaling during early oncogenesis. Cell. 2008:134:921-31.

17. Retsekova E, Reis-Filho JS, Jain RK, et al. Prognostic impact of ALDH1 in breast cancer: a story of stem cells and tumor microenvironment. Breast Cancer Res Treat. 2010;123:97-108.

18. Bednarz-Knoll N, Nastaly P, Zaczek A, et al. Oncotarget. 2015:29:26789-803.

19. Isfoss $B L$, Holmqvist $B$, Jernström $H$, et al. Women with familial risk for breast cancer have an increased frequency of aldehyde dehydrogenase expressing cells in breast ductules. BMC Clin Pathol. 2013;13:28.

20. Isfoss BL, Holmqvist B, Jernström $H$, et al. The absence of aldehyde dehydrogenase $1 \mathrm{A1}$-positive cells in benign mammary stroma is associated with risk factors for breast cancer. Breast Cancer (Dove Med Press). 2016;8:117-24.

21. Wakao S, Kitada M, Kuroda Y, et al. Multilineage-differentiating stressenduring (muse) cells are a primary source of induced pluripotent stem cells in human fibroblasts. Proc Natl Acad Sci U S A. 2011;108:9875-80.

22. Dezawa M. Muse cells provide the pluripotency of mesenchymal stem cells: direct contribution of muse cells to tissue regeneration. Cell Transplant. 2016;25:849-61.

23. Wake K. Perisinusoidal stellate cells (fat-storing cells, interstitial cells, lipocytes), their related structure in and around the liver sinusoids, and vitamin A-storing cells in extrahepatic organs. Int Rev Cytol. 1980;66:303-53.

24. Nordlinder H, Eriksson U, Busch C. Identification of extrahepatic stellate cells and the cell specific regulation of cellular retinol binding protein. In: distribution of cytoplasmic retinol- and retinoic acid-binding proteins: identification of an interstitial vitamin A-storing cell system. Doctoral thesis no. 284 (paper 5). Uppsala: Acta Universitatis Upsaliensis; 1991.

25. Friedman SL. Hepatic stellate cells: protean, multifunctional, and enigmatic cells of the liver. Physiol Rev. 2008;88:125-72.

26. Isfoss BL, Busch $\mathrm{C}$, Hermelin $\mathrm{H}$, et al. Stem cell marker-positive stellate cells and mast cells are reduced in benign-appearing bladder tissue in patients with urothelial carcinoma. Virchows Arch. 2014;464:473-88.

27. Olsson $H$, Jernström $H$, Alm P, et al. Proliferation of the breast epithelium in relation to menstrual cycle phase, hormonal use, and reproductive factors. Breast Cancer Res Treat. 1996:40:187-96.

28. Yang Z, Liu J, Liu H, et al. Isolation and characterization of SSEA3(+) stem cells derived from goat skin fibroblasts. Cell Reprogram. 2013;15:195-205.

29. Suzuki $Y$, Haraguchi $N$, Takahashi $H$, et al. SSEA-3 is a novel amplifyng cancer cell surface marker in colorectal cancers. Int J Oncol. 2012;42:161-7.

30. Chang WW, Lee CH, Lee P, et al. Expression of Globo $\mathrm{H}$ and SSEA3 in breast cancer stem cells and the involvement of fucosyl transferases 1 and 2 in Globo H synthesis. Proc Natl Acad Sci U S A. 2008;105:11667-72.

31. Johansson $\mathrm{O}$, Loman $\mathrm{N}$, Borg $\mathrm{A}$, et al. Pregnancy-associated breast cancer in BRCA1 and BRCA2 germline mutation carriers. Lancet. 1998;352:1359-60.

32. Kotsopoulos J, Lubinski J, Lynch HT, et al. Age at first birth and the risk of breast cancer in BRCA1 and BRCA2 mutation carriers. Breast Cancer Res Treat. 2007;105:221-8.

33. Jernström $H$, Leman $C$, Ghadirian $P$, et al. Pregnancy and risk of early breast cancer in carriers of BRCA1 and BRCA2. Lancet. 1999:354:1846-50.

34. Harvey JA, Santen RJ, Petroni GR, et al. Histologic changes in the breast with menopausal hormone therapy use: correlation with breast identity, estrogen receptor, progesterone receptor, and proliferation indices. Menopause. 2008;15:67-73.

35. Kinoshita K, Kuno S, Ishimine $\mathrm{H}$, et al. Therapeutic potential of adiposederived SSEA-3-positive muse cells for treating diabetic skin ulcers. Stem Cells Transl Med. 2015:4:146-55.

36. Hori E, Hayakawa Y, Hayashi T, et al. Mobilization of pluripotent multilineage-differentiating stress-enduring cells in ischemic stroke. J Stroke Cerebrovasc Dis. 2016;25:1473-81. 
37. Van Rossen E, Vander Borght $S$, van Grunsven LA, et al. Vinculin and cellular retinol-binding protein-1 are markers for quiescent and activated hepatic stellate cells in formalin-fixed paraffin embedded human liver. Histochem Cell Biol. 2009;131:313-24.

38. Coombs CC, Tallman MS, Levine RL. Molecular therapy for acute myeloid leukemia. Nat Rev Clin Oncol. 2016;13:305-18.

39. Morsing M, Klitgaard MC, Jafari A, et al. Evidence of two distinct functionally specialized fibroblast lineages in breast stroma. Breast Cancer Res. 2016;18:108.

Submit your next manuscript to BioMed Central and we will help you at every step:

- We accept pre-submission inquiries

- Our selector tool helps you to find the most relevant journal

- We provide round the clock customer support

- Convenient online submission

- Thorough peer review

- Inclusion in PubMed and all major indexing services

- Maximum visibility for your research

Submit your manuscript at www.biomedcentral.com/submit 